Claremont Colleges

Scholarship@ Claremont

All HMC Faculty Publications and Research

HMC Faculty Scholarship

$1-1-2001$

\title{
Transient Anomalous Diffusion in Poiseuille Flow
}

Marco Latini '01

Harvey Mudd College

Andrew J. Bernoff

Harvey Mudd College

\section{Recommended Citation}

MARCO LATINI and ANDREW J. BERNOFF (2001). Transient anomalous diffusion in Poiseuille flow. Journal of Fluid Mechanics, 441, pp 399-411 doi:10.1017/S0022112001004906

This Article is brought to you for free and open access by the HMC Faculty Scholarship at Scholarship @ Claremont. It has been accepted for inclusion in All HMC Faculty Publications and Research by an authorized administrator of Scholarship @ Claremont. For more information, please contact scholarship@cuc.claremont.edu. 


\title{
Transient anomalous diffusion in Poiseuille flow
}

\author{
By MARCO LATINI AND ANDREW J. BERNOFF \\ Harvey Mudd College, Claremont, CA 91711, USA
}

(Received 29 January 2001 and in revised form 13 March 2001)

We revisit the classical problem of dispersion of a point discharge of tracer in laminar pipe Poiseuille flow. For a discharge at the centre of the pipe we show that in the limit of small non-dimensional diffusion, $D$, tracer dispersion can be divided into three regimes. For small times $\left(t \ll D^{-1 / 3}\right)$, diffusion dominates advection yielding a spherically symmetric Gaussian dispersion cloud. At large times $\left(t \gg D^{-1}\right)$, the flow is in the classical Taylor regime, for which the tracer is homogenized transversely across the pipe and diffuses with a Gaussian distribution longitudinally. However, in an intermediate regime $\left(D^{-1 / 3} \ll t \ll D^{-1}\right)$, the longitudinal diffusion is anomalous with a width proportional to $D t^{2}$ and a distinctly asymmetric longitudinal distribution. We present a new solution valid in this regime and verify our results numerically. Analogous results are presented for an off-centre release; here the distribution width scales as $D^{1 / 2} t^{3 / 2}$ in the anomalous regime. These results suggest that anomalous diffusion is a hallmark of the shear dispersion of point discharges at times earlier than the Taylor regime.

\section{Introduction}

The basic mechanisms of tracer dispersion in laminar pipe Poiseuille flow were first studied by Taylor (1953) and extended by Aris (1956). They concluded that for sufficiently large time any localized initial configuration of tracer evolves to a symmetric Gaussian distribution moving with the mean speed of the flow and spreading longitudinally with an effective diffusion coefficient $D_{\text {eff }}=1 /(192 D)+D$, where $D$ is the non-dimensional diffusion coefficient. This behaviour is achieved only when sufficient transverse mixing has occurred, and even Taylor, in his experiments, noted that for moderate time a distinct asymmetry was observed. Lighthill (1966) addressed this discrepancy by describing the initial development of tracer dispersion before complete transverse mixing has occurred. Lighthill assumed the initial distribution of the tracer was radially uniform, and localized longitudinally as a $\delta$-function. He showed that the tracer distribution spreads longitudinally proportional to $t$, much faster than the characteristic diffusion width of $\sqrt{D t}$. This is perhaps the first observation of anomalous diffusion in the fluids literature.

In this paper, we first consider a $\delta$-function initial condition at the centre of the pipe. Using Lighthill's method we find an exact solution for the advection-diffusion equation, valid in the absence of the pipe's boundaries. This solution, where the width spreads anomalously proportional to $D t^{2}$, provides a useful approximation at times before a significant proportion of the tracer diffuses to the walls. From this solution we derive the distribution's longitudinal moments and useful approximations for the shape of the head and tail of the concentration profile. We verify our results 
through numerical simulation. For completeness, we also present results for an offcentre release, where the width spreads anomalously as $D^{1 / 2} t^{3 / 2}$ before the Taylor regime (cf. Elrick 1962; Rhines \& Young 1983). Finally, in the discussion, we present a dimensional analysis argument suggesting that transient anomalous diffusion is a feature of shear dispersion of a point discharge for laminar shear flows. In the Appendix we present analogous results for plane Poiseuille flow.

\subsection{Formulation of the problem}

Consider dispersion due to pipe Poiseuille flow,

$$
u(R, \theta, Z)=U_{0}\left(1-\frac{R^{2}}{a^{2}}\right),
$$

where $U_{0}$ is the centreline velocity and $a$ is the pipe radius. Let $C(R, \theta, Z, T)$ indicate the concentration of the passive scalar, being advected with the flow and diffusing with constant diffusivity $D^{*}$. In non-dimensional variables,

$$
t=\frac{U_{0}}{a} T, \quad r=\frac{R}{a}, \quad z=\frac{Z}{a},
$$

the system is governed by the advection-diffusion equation,

$$
\frac{\partial C}{\partial t}+\left(1-r^{2}\right) \frac{\partial C}{\partial z}=D\left(\frac{\partial^{2} C}{\partial r^{2}}+\frac{1}{r} \frac{\partial C}{\partial r}+\frac{1}{r^{2}} \frac{\partial^{2} C}{\partial \theta^{2}}+\frac{\partial^{2} C}{\partial z^{2}}\right),
$$

where $D=D^{*} / a U_{0}=1 / P e$ is a non-dimensional diffusion constant and $P e$ is the Péclet number. $D$ is quite small in typical applications (blood flow, sewage dispersion, etc). We consider a $\delta$-function initial condition at the centre of the pipe (the origin of our coordinate system) at $t=0$,

$$
C(r, \theta, z, 0)=\delta(z) \delta(\boldsymbol{r}),
$$

where $\delta(\boldsymbol{r})$ is a two-dimensional $\delta$-function at the origin in $(r, \theta)$. At the pipe's edge,

$$
\frac{\partial C}{\partial r}=0 \quad \text { at } \quad r=1,
$$

corresponding to no-flux boundaries. Integrating over the pipe's cross-section,

$$
\bar{C}(z, t)=\int_{\theta=0}^{2 \pi} \int_{r=0}^{1} C(r, \theta, z, t) r \mathrm{~d} r \mathrm{~d} \theta,
$$

yields the longitudinal concentration distribution of the tracer. Information on the shape of this distribution, such as whether it is peaked or asymmetric, its rate of movement and expansion, etc. can be obtained from the first few longitudinal moments, defined by

$$
M_{n}(t)=\int_{z=-\infty}^{\infty} z^{n} \bar{C}(z, t) \mathrm{d} z .
$$

Note that $M_{0}(t)=1$. In particular, the width of the distribution, $\sigma(t)$, is defined as

$$
\sigma(t)=\sqrt{M_{2}(t)-M_{1}^{2}(t)} .
$$

\subsection{Three regimes of shear dispersion}

Figure 1 illustrates the three stages of longitudinal dispersion that are observed in this problem. Initially the width scales diffusively $(\sigma \sim \sqrt{2 D t})$. In the anomalous regime, 


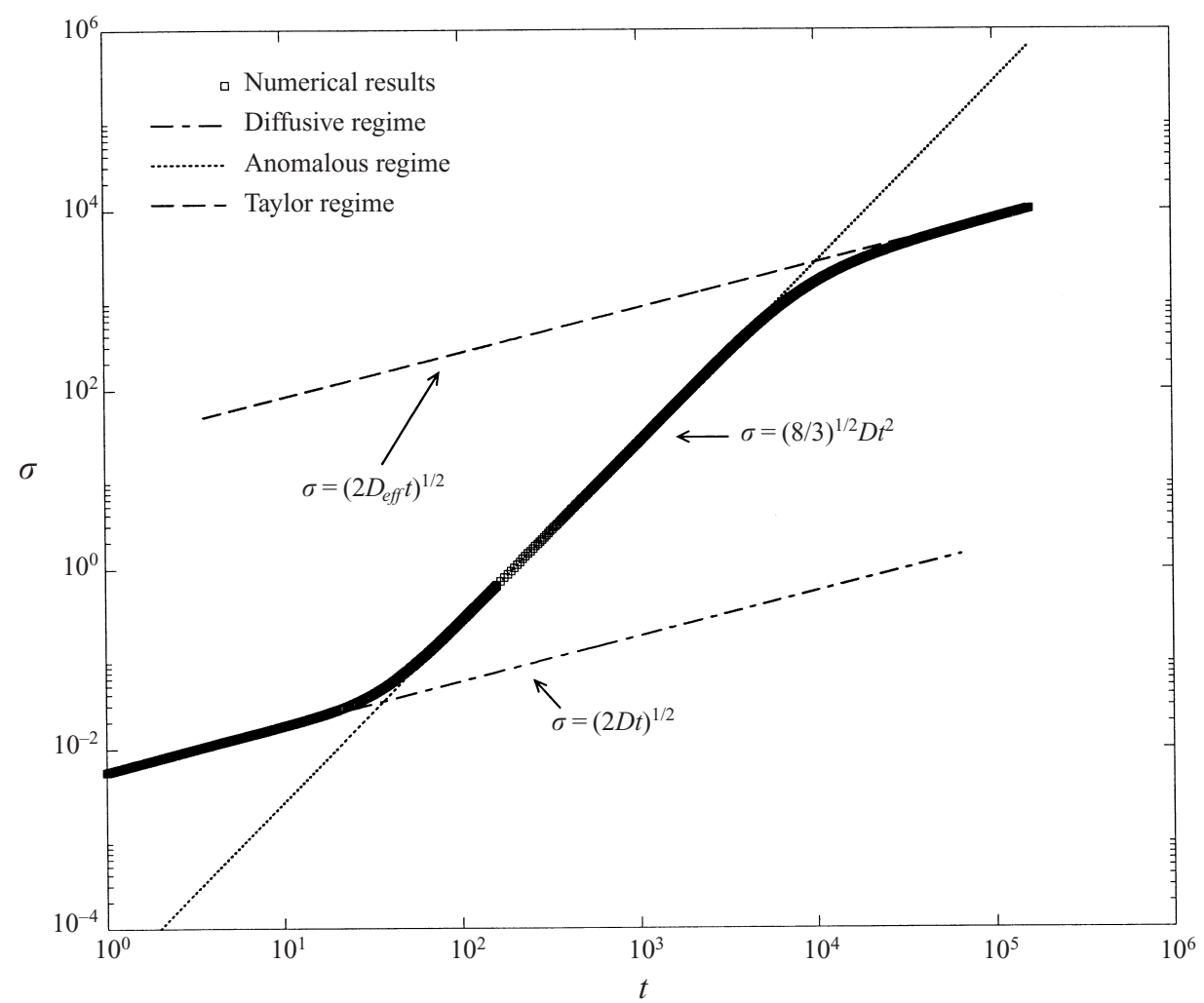

FIGURE 1. The three stages of longitudinal dispersion characterized by different scaling laws of $\sigma$. The results are for $D=1.625 \times 10^{-5}$.

the width scales in a superdiffusive fashion $\left(\sigma \sim \sqrt{8 / 3} D t^{2}\right)$. Finally, in the Taylor regime, the width again scales diffusively, but with a much larger diffusion coefficient $\left(\sigma \sim \sqrt{2 D_{\text {eff }} t}\right)$, where $D_{\text {eff }}=1 /(192 D)+D \dagger$. Let us examine these three regimes.

Diffusive regime $\left(t \ll D^{-1 / 3}\right)$ : Initially, dispersion due to diffusion dominates over the effects of shear (figure $2 a$ ). The longitudinal displacement due to diffusion $O(\sqrt{D t})$ is large compared to the relative longitudinal displacement of two particles, one at the centreline, and a second that has diffused transversely a distance $O(\sqrt{D t})$. These particles become separated by a distance $O\left(D t^{2}\right)$ due to the parabolic shear. Comparing the two longitudinal displacements, we obtain the range of validity cited above. At leading order, the longitudinal distribution is a Gaussian, advected with centreline velocity

$$
\bar{C}(z, t) \approx \frac{\exp \left(-(z-t)^{2} / 4 D t\right)}{\sqrt{4 \pi D t}}
$$

as is illustrated in figure $2(d)$.

Anomalous regime $\left(D^{-1 / 3} \ll t \ll D^{-1}\right)$ : In this regime the displacement due to tracer diffusing transversely and being sheared longitudinally dominates over the longitudinal diffusion. However, the majority of the tracer has not yet interacted

$\dagger$ Note that our definition of $D$ (and consequently of $D_{\text {eff }}$ ) differs from Aris' definition as we have non-dimensionalized on the midline velocity, $U_{0}$, not the average velocity $U_{m}=U_{0} / 2$. 

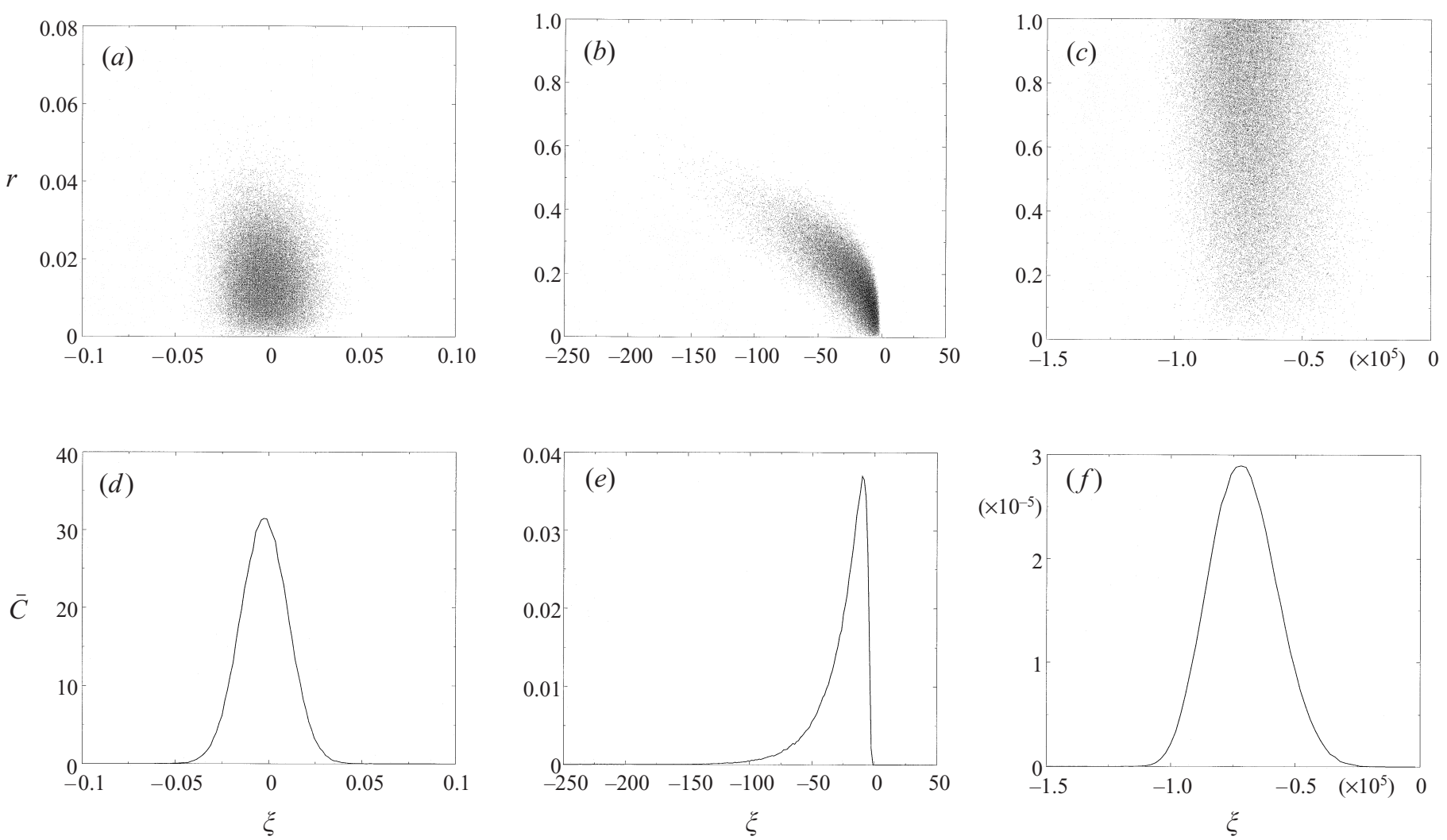

3
$\vdots$
$\vdots$
$\vdots$
$\vdots$
$\vdots$
$\vdots$
1
0
0
0
0
0
0

FIGURE 2. The three dispersion regimes observed in this problem. $(a-c)$ The particles are being advected and dispersing in the pipe, plotted as a function of the axial position $\xi=z-t$ and of the radius. $(d-f)$ The corresponding longitudinal distribution functions. The results are obtained for $D=8 \times 10^{-6}$. $(a, d)$ The diffusive regime at $t=10$. Note the Gaussian distribution. $(b, e)$ The anomalous regime at $t=1250$. Note the asymmetric and peaked distribution of the concentration profile. $(c, f)$ The Taylor regime at $t=150000$. The particles have diffused across the entire pipe and the distribution is a symmetric Gaussian. 
with the pipe's boundary. In this regime, the distribution is distinctly asymmetric (figure $2 b, e$ ) and is advected with the pipe's centreline velocity.

Taylor regime $\left(D^{-1} \ll t\right)$ : In the classic Taylor regime, the particles have become uniformly distributed over the cross-section of the pipe (figure $2 c, f$ ). The longitudinal distribution is again a Gaussian

$$
\bar{C}(z, t) \approx \frac{\exp \left(-\left(z-\frac{t}{2}\right)^{2} / 4 D_{\text {eff }} t\right)}{\sqrt{4 \pi D_{\text {eff }} t}}
$$

moving longitudinally with the average speed, and where $D_{\text {eff }}=1 /(192 D)+D$.

The Taylor regime of dispersion has received the greatest attention in the literature; Taylor (1953) argued that after the tracer has completely mixed across the tube diameter, it diffuses longitudinally as a Gaussian with an effective diffusion coefficient. Amazed by this "remarkable" result he then verified it experimentally. Aris (1956) improved Taylor's approximation of the effective diffusive coefficient, finding that $D_{\text {eff }}=1 /(192 D)+D$; he also first introduced longitudinal moments to characterize the tracer distribution. Chatwin (1970), noting the asymmetry of the tracer distribution at earlier times, investigated the approach to a Gaussian distribution by incorporating asymmetric corrections. Taylor's theory has also been extended to cover a great variety of transport problems well beyond straight pipes and unidirectional flows. Frankel \& Brenner (1989) gave a solid foundation to this generalized version of Taylor transport theory with an asymptotic expansion similar to the one developed by Chatwin for the classical theory. Another approach was taken by Gill \& Sankarasubramanian (1970) who showed that an advection-diffusion equation with a time-dependent diffusion coefficient could exactly reproduce the centroid and width of the concentration for all time. Smith (1981) improved their idea by using a delay-diffusion equation, capable of describing the asymmetry of the distribution and reproducing the skewness exactly. A more complete history can be found in the review by Young \& Jones (1991).

All these methods depend heavily on expanding the transverse tracer distribution in an eigenfunction expansion, in particular Bessel functions for a circular pipe. While this is valid and appropriate in the limit of complete transverse mixing, we will concentrate on earlier times when the scalar has not yet strongly interacted with the pipe boundary, and a free-space expansion is more appropriate.

The diffusive limit was discussed by Chatwin $(1976,1977)$ based on earlier work by Saffman (1960). Chatwin noted that for small values of time longitudinal diffusion could not be neglected; he developed a Gaussian approximation, multiplied by a Taylor series in time, valid as $t \rightarrow 0$.

Lighthill (1966) first studied the anomalous regime; he noticed that for large arteries the flow times were too small for the theory of Taylor to apply. Lighthill assumed the initial distribution of the tracer was radially uniform, and localized longitudinally as a $\delta$-function. In the absence of diffusion, he argued that the longitudinal distribution of the tracer would be a uniform-height finite-width step, with a support between the initial point of release (corresponding to tracer at the stagnant edge of the pipe) and the head of the distribution which moves with the centreline velocity. This solution spreads ballistically, with a width proportional to $t$. Lighthill then found an exact solution for the concentration incorporating transverse diffusion, but ignoring longitudinal diffusion and interactions with the pipe's boundary, in terms of a Fourier transform. He found that the sharp transition at the head of the uniform step turned into a transition layer which spread at a rate proportional to $D t^{2}$, foreshadowing the results presented here. His solution is valid for times before the tracer interacts strongly with the tube wall, i.e. before the Taylor regime. 
Subsequent authors have tried to take a more unified approach to the problem, allowing a description of the solution valid for all times. Stokes \& Barton (1990) developed a description based on a Laplace transform in time and a Fourier transform in space, yielding an eigenfunction problem for the radial dependence. These ideas were extended by Phillips \& Kaye $(1996,1997)$. Most relevant to the problem studied here is an expansion in Phillips \& Kaye (1997) done for a parabolic profile in an infinite domain; they found an infinite expansion in terms of the product of Laguerre polynomials and Gaussians. They used this expansion to recover Lighthill's expansion at the head of the distribution. This method can be used with the $\delta$-function initial condition discussed here, and Phillips \& Kaye give the first term of the head expansion. Our results give complete expansions for both the head and the tail of the radially averaged concentration. In addition, our derivation is more compact and avoids the radial eigenfunction expansion.

The related problem for linear shear has been well studied; Elrick (1962) gives the exact solution, a Gaussian, for a $\delta$-function release. Related results and extensions can also be found in Foister \& Van De Ven (1984), Rhines \& Young (1983), Liron \& Rubinstein (1984) and references therein. Brunet \& Haynes (1995) found a solution analogous to Lighthill's solution for plane Poiseuille flow, again with a radially uniform initial condition. Pasmanter (1985) found a variety of exact solutions for advection-diffusion equations including rederiving Lighthill's solution.

Recently there has been interest in problems exhibiting anomalous diffusion, where the variance of the concentration distribution, $\sigma^{2}(t) \propto t^{\gamma}$, spreads with a non-diffusive exponent $(\gamma \neq 1)$ (Young \& Jones 1991; Weeks, Urbach \& Swinney 1996; ben Avraham, Leyvraz \& Redner 1992; Jones 1994). Weeks et al. (1996) give a Levy flight model for these behaviours; they describe superdiffusive $(\gamma>1)$ and ballistic behaviour $(\gamma=2)$ associated with a collection of particles each moving with its own velocity. The behaviour in the anomalous regime here is superballistic, with particles separating faster than the ballistic case as observed in the particle model of ben Avraham et al. (1992). Though this behaviour is transient, its appearance in this model suggests that anomalous diffusion due to differential shear may be a common feature observable at early times in fluid experiments.

Our numerical results are obtained using the split-step Monte Carlo method developed for advection-diffusion equations by Lingevitch \& Bernoff (1994); this method is particularly robust for small values of the diffusion constant.

\section{The anomalous regime}

The advection-diffusion equation (1.2) is solved with an approach similar to the one taken by Lighthill (1966). This yields an approximation that is valid for both the initial diffusive regime and the anomalous regime.

We Fourier transform the advection-diffusion equation in the $z$-direction; define

$$
\hat{C}(r, \theta, k, t)=\int_{-\infty}^{\infty} C(r, \theta, z, t) \mathrm{e}^{-\mathrm{i} k z} \mathrm{~d} z,
$$

then

$$
\hat{C}_{t}+\mathrm{i} k\left(1-r^{2}\right) \hat{C}=D\left(\hat{C}_{r r}+\frac{\hat{C}_{r}}{r}+\frac{\hat{C}_{\theta \theta}}{r^{2}}\right)-D k^{2} \hat{C}, \quad \hat{C}(r, \theta, k, 0)=\delta(\boldsymbol{r}) .
$$


Now substituting $\hat{C}(r, \theta, k, t)=\hat{B}(r, k, t) \exp \left(-\left(\mathrm{i} k+k^{2} D\right) t\right)$ yields

$$
\hat{B}_{t}-\mathrm{i} k r^{2} \hat{B}=D\left(\hat{B}_{r r}+\frac{\hat{B}_{r}}{r}\right) .
$$

Following Lighthill, we look for a solution of the form $\hat{B}(r, k, t)=f(k, t) \exp \left(r^{2} g(k, t)\right)$, which yields two differential equations:

$$
f_{t}=4 D f g, \quad g_{t}=4 D g^{2}+\mathrm{i} k .
$$

Solving the Riccati equation for $g(k, t)$ yields the general solution:

$$
g(k, t)=-\frac{q}{4 D}\left(\frac{\mathrm{e}^{q t}-B \mathrm{e}^{-q t}}{\mathrm{e}^{q t}+B \mathrm{e}^{-q t}}\right), \quad f(k, t)=\frac{H}{\mathrm{e}^{q t}+B \mathrm{e}^{-q t}},
$$

where $q=\sqrt{-4 D \mathrm{ik}}$.

Lighthill chose $B=1$, corresponding to an initial condition that is uniform transversely to the pipe and a $\delta$-function longitudinally $[\hat{C}(r, \theta, k, 0)=1]$. If we let $B=-1$,

$$
g(k, t)=-\frac{q}{4 D} \operatorname{coth} q t, \quad f(k, t)=\frac{H}{2 \sinh q t},
$$

as $t \rightarrow 0$, this solution approaches a $\delta$-function initial condition at the origin,

$$
\hat{C}(r, \theta, k, t) \rightarrow \frac{H \exp \left(-r^{2} / 4 D t\right)}{2 q t} .
$$

We choose $H=q / 2 \pi D$ corresponding to the unit mass.

This yields an exact solution to the advection-diffusion problem (2.1):

$$
\hat{C}(r, \theta, k, t)=\frac{q}{4 \pi D} \frac{\exp \left(-q r^{2} /(4 D \tanh q t)\right)}{\sinh q t} \exp \left(-\left(\mathrm{i} k+k^{2} D\right) t\right) .
$$

\subsection{Moments}

Integrating (2.2) over the transverse cross-section yields the Fourier transform of the longitudinal distribution function:

$$
\hat{\bar{C}}(k, t)=\int_{\theta=0}^{2 \pi} \int_{r=0}^{\infty} \hat{C}(r, \theta, k, t) r \mathrm{~d} r \mathrm{~d} \theta=\operatorname{sech}(q t) \exp \left(-\left(\mathrm{i} k+k^{2} D\right) t\right) .
$$

The $n$th moment of the averaged concentration (cf. (1.5)), $M_{n}(t)$, is given by

$$
M_{n}(t)=\left.(\mathrm{i})^{n} \frac{\partial^{n} \hat{\bar{C}}}{\partial k^{n}}\right|_{k=0} .
$$

Note that $M_{0}(t)=1$ due to conservation of mass. Also

$$
\begin{gathered}
M_{1}(t)=-2 D t^{2}+t, \quad M_{2}(t)=\frac{20}{3} D^{2} t^{4}-4 D t^{3}+t^{2}+2 D t, \\
M_{3}(t)=-\frac{488}{15} D^{3} t^{6}+20 D^{2} t^{5}-6 D t^{4}+\left(1-12 D^{2}\right) t^{3}+6 D t^{2}, \\
M_{4}(t)=\frac{4432}{21} D^{4} t^{8}-\frac{1952}{15} D^{3} t^{7}+40 D^{2} t^{6}+\left(80 D^{3}-8 D\right) t^{5}+\left(1-48 D^{2}\right) t^{4}+12 D t^{3}+12 D^{2} t^{2} .
\end{gathered}
$$

The variance $\sigma^{2}(t)$ can now be calculated (assuming $M_{0}(t)=1$ ):

$$
\sigma^{2}(t)=M_{2}(t)-M_{1}^{2}(t)=\frac{8}{3} D^{2} t^{4}+2 D t .
$$


Note that for the diffusive regime $\left(t \ll D^{-1 / 3}\right) \sigma \sim \sqrt{2 D t}$, while in the anomalous regime $\left(t \gg D^{-1 / 3}\right) \sigma \sim \sqrt{8 / 3} D t^{2}$. This result is verified numerically in figure 1 , and is clearly valid until $t \approx O\left(D^{-1}\right)$ when the tracer begins to interact with the pipe's boundary.

We can also evaluate the skewness and the kurtosis in the anomalous regime:

$$
\begin{gathered}
S k=\lim _{t \rightarrow \infty} \frac{M_{3}(t)-3 M_{1}(t) M_{2}(t)+2 M_{1}^{3}(t)}{\sigma^{3}(t)}=-\frac{4 \sqrt{6}}{5} \approx-1.959591794 \\
K u=\lim _{t \rightarrow \infty} \frac{-3 M_{1}^{4}(t)+6 M_{1}^{2}(t) M_{2}(t)-4 M_{1}(t) M_{3}(t)+M_{4}(t)}{\sigma^{4}(t)}=\frac{309}{35} \approx 8.828571429 .
\end{gathered}
$$

The negative value of the skewness indicates an asymmetric profile with a tail to the left of the maximum. The large positive kurtosis indicates a profile with a much narrower peak than a Gaussian distribution (for which $K u=3$ ).

\subsection{Longitudinal concentration profile}

Computing the inverse Fourier transform of (2.3) yields the longitudinal concentration profile:

$$
\bar{C}(z, t)=\frac{1}{2 \pi} \int_{-\infty}^{\infty} \hat{\bar{C}}(k, t) \mathrm{e}^{\mathrm{i} k z} \mathrm{~d} k=\frac{1}{2 \pi} \int_{-\infty}^{\infty} \operatorname{sech}(q t) \mathrm{e}^{-\left(\mathrm{i} k+k^{2} D\right) t} \mathrm{e}^{\mathrm{i} k z} \mathrm{~d} k .
$$

The problem simplifies if we choose similarity variables,

$$
\ell=4 D k t^{2}, \quad \eta=\frac{z-t}{4 D t^{2}},
$$

in a reference frame moving with the centreline velocity. Then

$$
\begin{aligned}
\bar{C}(\eta, t) & =\frac{1}{8 \pi D t^{2}} \int_{-\infty}^{\infty} \operatorname{sech}(\sqrt{-\mathrm{i} \ell}) \mathrm{e}^{\mathrm{i} / \eta} \exp \left(-\frac{\ell^{2}}{16 D t^{3}}\right) \mathrm{d} \ell \\
& \sim \frac{1}{8 \pi D t^{2}} \int_{-\infty}^{\infty} \operatorname{sech}(\sqrt{-\mathrm{i} \ell}) \mathrm{e}^{\mathrm{i} \ell \eta} \mathrm{d} \ell+O\left(\frac{1}{D^{2} t^{5}}\right) .
\end{aligned}
$$

For $t \gg D^{-1 / 3}$, the anomalous regime, we neglect the term $\exp \left(-\ell^{2} / 16 D t^{3}\right)$, which is associated with longitudinal diffusion.

This integral can be evaluated with contour integration. The singularities of the integrand are simple poles in the lower half-plane along the negative imaginary axis at $\alpha_{n}=-\mathrm{i} \pi^{2}\left(n+\frac{1}{2}\right)^{2}$. For $\eta>0$ the integral can be closed in the upper half-plane where the function is analytic, therefore $\bar{C}(\eta, t)=0$. For $\eta<0$ we close the contour in the lower half-plane picking up the residue from each pole:

$$
\begin{aligned}
\bar{C}(\eta, t) & \sim \frac{\mathrm{i}}{4 D t^{2}} \sum_{n=0}^{\infty} \operatorname{Res}\left[\operatorname{sech}(\sqrt{-\mathrm{i} \ell}) \mathrm{e}^{\mathrm{i} \ell \eta} ; \alpha_{n}\right] \\
& =\frac{\pi}{4 D t^{2}} \sum_{n=0}^{\infty}(-1)^{n}(2 n+1) \exp \left((2 n+1)^{2} \pi^{2} \eta / 4\right) .
\end{aligned}
$$

This series converges for $\eta<0$ and is a good approximation for $|\eta| \gg 1$.

For $0<-\eta \ll 1$ we find a different approximation that can account for the peak in the concentration profile. Note that if we differentiate with respect to $x$ the Jacobi 


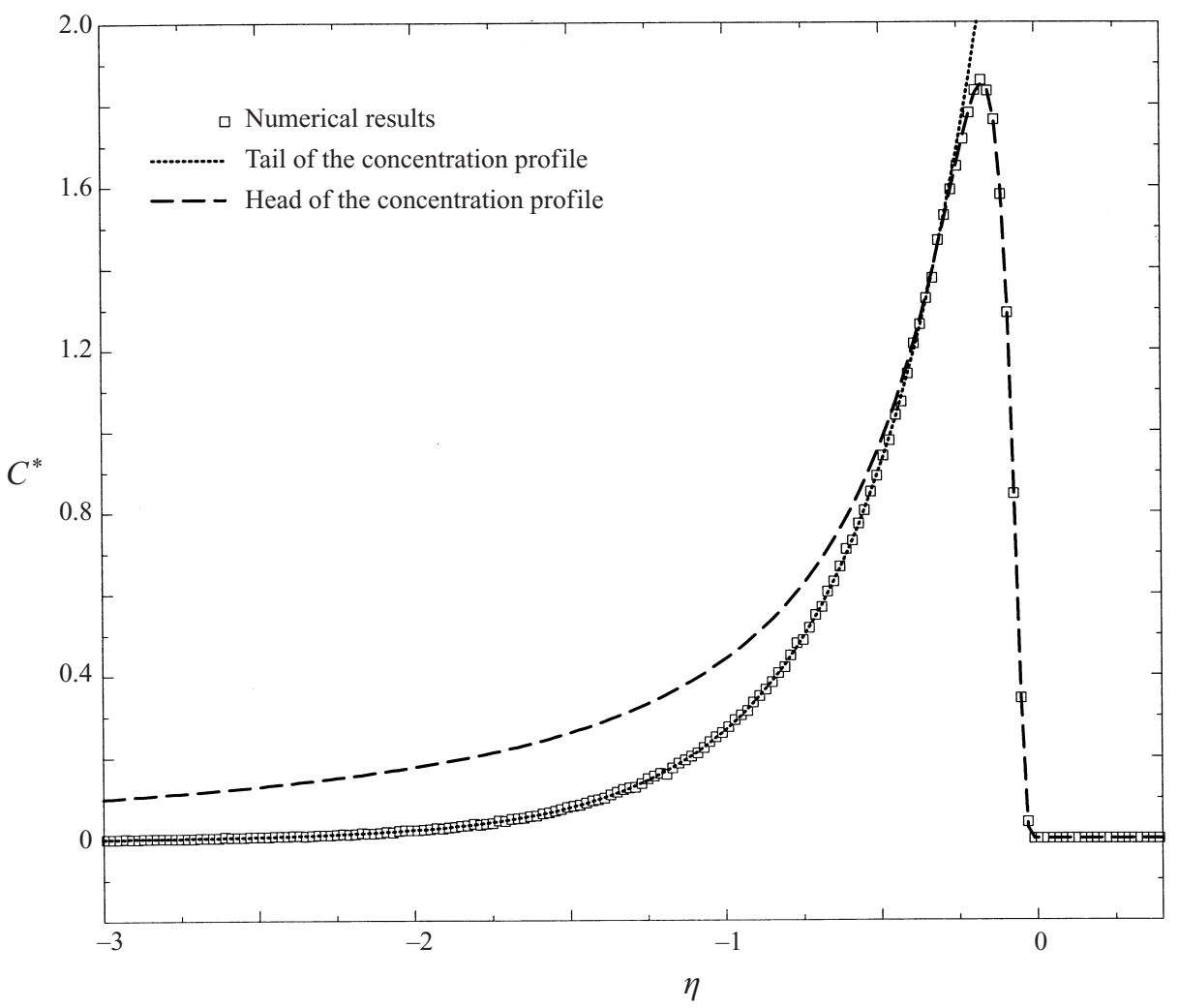

FIGURE 3. The concentration profile at time $t=1250$ with the first term of the head and tail approximations (2.9) and (2.8). The numerical results are obtained for $D=8 \times 10^{-6}$. Here $\eta=(z-t) / 4 D t^{2}$ and $C^{*}(\eta, t)=4 D t^{2} \bar{C}(\eta, t)$ is the coordinate system. The approximations cross at $\eta=-1 / \pi$.

theta function identity found in Bellman (1961, p. 10)

$$
\sum_{n=-\infty}^{\infty} \exp \left(-t(x+n)^{2}\right)=\sqrt{\frac{\pi}{t}} \sum_{k=-\infty}^{\infty} \exp \left(2 \pi \mathrm{i} k x-\left(\pi^{2} k^{2} / t\right)\right)
$$

and choose appropriate values for the constants, we are able to recover (2.8) from the left-hand side. The right-hand side yields

$$
\bar{C}(\eta, t)=\frac{\pi}{4 D t^{2}} \frac{1}{(-\pi \eta)^{3 / 2}} \sum_{n=0}^{\infty}(-1)^{n}(2 n+1) \exp \left((2 n+1)^{2} / 4 \eta\right) .
$$

This result has information about the head of the concentration of passive tracer. Note the first term of this expansion can be deduced from equation (3.7) in Phillips $\&$ Kaye (1997). Figure 3 displays the longitudinal concentration profile with the head and tail approximations.

\subsection{Off-centre point discharge}

We now consider a point discharge release at an arbitrary location along the crosssection of the pipe. Note that it is always possible to rotate the Cartesian coordinate system so that the release occurs at some positive value $b$, for $0<b<1$ along the $y$-axis. If we rescale in the neighbourhood of the point release, $X=x / \sqrt{D}$, $Y=(y-b) / \sqrt{D}, Z=\left(z-\left(1-b^{2}\right) t\right) / \sqrt{D}, T=t$, then equation (1.2) for $C(X, Y, Z, T)$ 


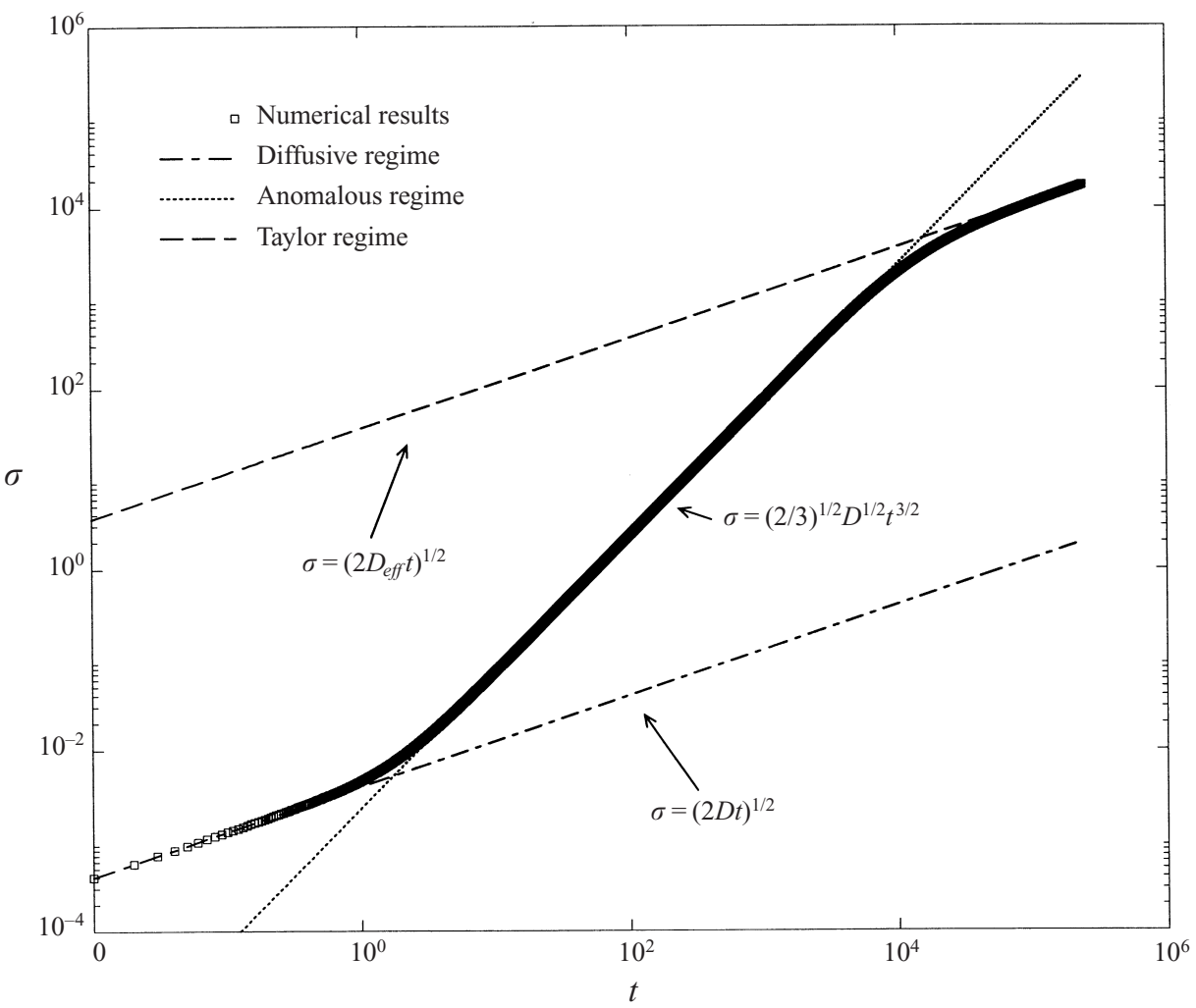

FIGURE 4. The three stages of longitudinal dispersion characterized by different scaling laws of $\sigma$ for a release of tracer at $b=1 / 2$. The results are for $D=8 \times 10^{-6}$.

becomes

$$
\frac{\partial C}{\partial T}-2 b Y \frac{\partial C}{\partial Z}=\nabla^{2} C+O\left(\sqrt{D} \frac{\partial C}{\partial Z}\left(X^{2}+Y^{2}\right)\right)
$$

This equation has an exact solution (cf. Elrick 1962; Rhines \& Young 1983)

$C(X, Y, Z, T)=\frac{1}{8 \pi D T \sqrt{\pi D T\left(1+b^{2} T^{2} / 3\right)}} \exp \left(-\frac{1}{4 T}\left(\frac{(Z+b Y T)^{2}}{1+b^{2} T^{2} / 3}+Y^{2}+X^{2}\right)\right)$.

Integrating this result over the cross-section yields the longitudinal concentration distribution

$$
\bar{C}(Z, T)=\frac{\sqrt{3}}{2 \sqrt{\pi D T}} \frac{1}{\sqrt{4 b^{2} T^{2}+3}} \exp \left(-\frac{3}{4 T} \frac{Z^{2}}{4 b^{2} T^{2}+3}\right),
$$

a Gaussian distribution in the longitudinal direction. The variance,

$$
\sigma^{2}(t)=\frac{8}{3} D b^{2} t^{3}+2 D t,
$$

is again anomalous and superdiffusive. This approximation is valid when the tracer has only diffused a small distance radially, for $t \ll D^{-1}$. This indicates that even in the presence of an off-centre tracer discharge we observe a transient anomalous regime. Figure 4 displays the three distinct regimes when the tracer is released for $b=1 / 2$. 


\section{Discussion}

Anomalous diffusion due to differential shear is clearly a feature of dispersion in Poiseuille flow, before the tracer interacts strongly with the pipe's wall. Our results suggest that two hallmarks of a point discharge at the centre of the pipe are a width $\sigma \propto D t^{2}$ and a strongly peaked asymmetric longitudinal distribution. An off-centre point discharge still produces an anomalous spread with a width proportional to $\sigma \propto D^{1 / 2} t^{3 / 2}$ and a symmetric Gaussian distribution.

These results can be understood on dimensional grounds, following an argument given by Rhines \& Young 1983. Consider a point discharge in a Lagrangian frame moving with a parallel shear flow that scales locally as $(\Delta y)^{n}$ where $\Delta y$ is the transverse displacement. After a time $t$, the tracer will have diffused transversely a distance proportional to $\sqrt{D t}$. Consequently, the difference in velocity, $\Delta v$, between tracer in the centre and the limb of the distribution will scale as $\Delta v \propto(D t)^{n / 2}$. Finally, the width of the distribution will scale as $\sigma \propto(\Delta v) t \propto D^{n / 2} t^{n / 2+1}$, in agreement with the Poiseuille flow results for release on the centreline $(n=2)$ and away from the centreline $(n=1)$ given above. Note that as $t$ approaches $1 / D$ from below a transition is seen to the Taylor regime. At this transition the width $\sigma \propto 1 / D \gg 1$, suggesting that the anomalous regime is relevant for large distribution widths that should be easily observable in experimental settings.

This research suggests that transient anomalous diffusion, at times before the Taylor regime, is a robust feature of shear dispersion in laminar flows. The solutions presented here can be used to describe the initial dispersion of a point discharge in either a local quadratic or linear shear.

The authors would like to thank Bill Young, Neil Balmforth, Andrea Bertozzi and Tom Witelski for enlightening conversations. Parts of this research were conducted at Duke University and the WHOI GFD Summer School. This research was supported by NSF grants DMS-9971969 and DMS-0074049. The authors also wish to thank the referees for their careful and constructive criticism.

\section{Appendix. Application to Poiseuille flow in a channel}

In this appendix we present results for plane Poiseuille flow using the same techniques. The system is governed by the advection-diffusion equation in two dimensions with a $\delta$-function initial condition at the channel's centre:

$$
\frac{\partial C}{\partial t}+\left(1-y^{2}\right) \frac{\partial C}{\partial z}=D \nabla^{2} C, \quad C(y, z, 0)=\delta(y) \delta(z),
$$

and no-flux boundary conditions at $y= \pm 1$.

The Fourier-transformed concentration

$$
\hat{C}(y, k, t)=\int_{-\infty}^{\infty} C(y, z, t) \mathrm{e}^{-\mathrm{i} k z} \mathrm{~d} z
$$

satisfies

$$
\hat{C}(y, k, t)=\frac{\sqrt{q}}{2 \sqrt{\pi D}} \frac{\exp \left(q y^{2} \operatorname{coth} q t / 4 D\right)}{\sqrt{\sinh q t}} \exp \left(-\left(\mathrm{i} k+k^{2} D\right) t\right) .
$$

Moments are obtained by integrating (A 2) over the width of the channel,

$$
\hat{\bar{C}}(k, t)=\int_{-\infty}^{\infty} \hat{C}(y, k, t) \mathrm{d} y=\exp \left(-\left(\mathrm{i} k+k^{2} D\right) t\right) \sqrt{\operatorname{sech} q t},
$$


and by differentiating and evaluating at 0 (cf. (2.4)). This yields

$$
\begin{gathered}
M_{1}(t)=-D t^{2}+t, \quad M_{2}(t)=\frac{7}{3} D^{2} t^{4}-2 D t^{3}+t^{2}+2 D t, \\
M_{3}(t)=-\frac{139}{15} D^{3} t^{6}+7 D^{2} t^{5}-3 D t^{4}+\left(1-6 D^{2}\right) t^{3}+6 D t^{2}, \\
M_{4}(t)=\frac{5473}{105} D^{4} t^{8}-\frac{556}{15} D^{3} t^{7}+14 D^{2} t^{6}+\left(28 D^{3}-4 D\right) t^{5}+\left(1-24 D^{2}\right) t^{4}+12 D t^{3}+12 D^{2} t^{2}, \\
\sigma^{2}(t)=M_{2}(t)-M_{1}^{2}(t)=\frac{4}{3} D^{2} t^{4}+2 D t, \\
S k=\lim _{t \rightarrow \infty} \frac{M_{3}(t)-3 M_{1}(t) M_{2}(t)+2 M_{1}^{3}(t)}{\sigma^{3}(t)}=-\frac{8 \sqrt{3}}{5}=-2.771281293, \\
K u=\lim _{t \rightarrow \infty} \frac{-3 M_{1}^{4}(t)+6 M_{1}^{2}(t) M_{2}(t)-4 M_{1}(t) M_{3}(t)+M_{4}(t)}{\sigma^{4}(t)}=\frac{513}{35}=14.65714286 .
\end{gathered}
$$

Computing the inverse Fourier transform of (A 3) yields

$$
\bar{C}(z, t)=\frac{1}{2 \pi} \int_{-\infty}^{\infty} \hat{\bar{C}}(k, t) \mathrm{e}^{\mathrm{i} k z} \mathrm{~d} k=\frac{1}{2 \pi} \int_{-\infty}^{\infty} \sqrt{\operatorname{sech} q t} \exp \left(-\left(\mathrm{i} k+k^{2} D\right) t\right) \mathrm{e}^{\mathrm{i} k z} \mathrm{~d} k .
$$

Using similarity variables $\ell=4 D k t^{2}, \eta=z-t / 4 D t^{2}$ and again neglecting the term $\mathrm{e}^{-k^{2} D t}$ in the anomalous regime $\left(D^{-1 / 3} \ll t \ll D^{-1}\right)$ yields

$$
\bar{C}(\eta, t)=\frac{1}{8 \pi D t^{2}} \int_{-\infty}^{\infty} \frac{\mathrm{e}^{\mathrm{i} \ell \eta}}{\sqrt{\cosh \sqrt{-\mathrm{i} \ell}}} \mathrm{d} \ell .
$$

This integral can be approximated via contour integration. The singularities of the integrand all lie on the negative real axis so $\bar{C}(\eta, t)=0$ for $\eta>0$. The square root creates a branch at each singular point. However, we can pair the singularities and let the branch cuts connect consecutive singularities. In this way the integration contour will evaluate to loop integrals around the pairs of singularities. As the integrand is decaying exponentially we approximate the integral by a loop integral around the first pair of singularities, yielding

$$
\bar{C}(\eta, t) \sim \frac{1}{4 D t^{2}} \frac{\exp \left(\frac{\pi^{2}}{4} \eta\right)}{\sqrt{-\eta}} \text { for }-\eta \gg 1 .
$$

For $0<-\eta \ll 1$ steepest descents yields

$$
\bar{C}(\eta, t) \sim \frac{1}{8 \sqrt{2 \pi} D t^{2}} \frac{1}{(-\eta)^{3 / 2}} \mathrm{e}^{1 /(16 \eta)} \quad \text { as } \quad \eta \rightarrow 0^{-} .
$$

\section{REFERENCES}

ARIS, R. 1956 On the dispersion of a solute in a fluid flowing through a tube. Proc. R. Soc. Lond. A 235, 67-77.

ben Avraham, D., Leyvraz, F. \& Redner, S. 1992 Superballistic motion in a random walk shear flow. Phys. Rev. A 45, 2315-2319.

Bellman, R. 1961 A Brief Introduction to Theta Functions. Rinehart and Winston, New York.

BRunet, G. \& HaYnes, P. H. 1995 The nonlinear evolution of disturbances to a parabolic jet. J. Atmos. Sci. 52, 464- 477. 
Chatwin, P. C. 1970 The approach to normality of the concentration of a solute in a solvent flowing along a straight pipe. J. Fluid Mech. 43, 321-352.

Chatwin, P. C. 1976 The initial dispersion of contaminant in poiseuille flow and the smoothing of the snout. J. Fluid Mech. 77, 593-602.

Chatwin, P. C. 1977 The initial development of longitudinal dispersion in straight tubes. J. Fluid Mech. 80, 33-48.

Elrick, D. E. 1962 Source functions for diffusion in uniform shear flow. Aust. J. Phys. 15, 283-288.

Foister, R. T. \& VAN De Ven, T. G. M. 1984 Diffusion of Brownian particles in shear flows. J. Fluid Mech. 143, 1-21.

Frankel, I. \& Brenner, H. 1989 On the foundations of generalized Taylor dispersion theory. J. Fluid Mech. 204, 97-119.

Gill, W. N. \& Sankarasubramanian, R. 1970 Exact analysis of unsteady convective diffusion. Proc. R. Soc. Lond. A 316, 341-350.

Jones, S. W. 1994 Chaotic advection and dispersion. Physica. D 76, 55-69.

Lighthill, M. J. 1966 Initial development of diffusion in poiseuille flow. J. Inst. Maths Appl. 2, 97-108.

LingevitCH, J. F. \& BeRnOFF, A. J. 1994 Advection of a passive scalar by a vortex couple in the small diffusion limit. J. Fluid Mechs 270, 219-249.

Liron, N. \& Rubinstein, J. 1984 Calculating the fundamental solution to linear convection-diffusion problems. SIAM J. Appl. Maths 44, 493-511.

Pasmanter, R. A. 1985 Exact and approximate solutions of the convection-diffusion equation. Q. J. Mech. Appl. Maths 38, 1-26.

Phillips, C. G. \& KaYE, S. R. 1996 A uniformly asymptotic approximation for the development of shear dispersion. J. Fluid Mech. 329, 413-443.

Phillips, C. G. \& KaYe, S. R. 1997 The initial transient of concentration during the development of Taylor dispersion. Proc. R. Soc. Lond. A 453, 2669-2688.

RHINES, P. B. \& YounG, W. R. 1983 How rapidly is a passive scalar mixed within closed streamlines? J. Fluid Mech. 123, 133-145.

SaffMAn, P. G. 1960 On the effect of the molecular diffusivity in turbulent diffusion. J. Fluid Mech. 8, 273-283.

Sмith, R. 1981 The importance of discharge siting upon contaminant dispersion in narrow rivers and estuaries. J. Fluid Mech. 108, 43-53.

Stokes, A. N. \& Barton, N. G. 1990 The concentration distribution produced by shear dispersion of solute in poiseuille flow. J. Fluid Mech. 210, 201-221.

TAYLOR, G. I. 1953 Dispersion of a soluble matter in solvent flowing slowly through a tube. Proc. R. Soc. Lond. A 219, 186-203.

Weeks, E. R., Urbach, J. S. \& Swinney, H. L. 1996 Anomalous diffusion in asymmetric random walks with a quasi-geostrophic flow example. Physica D 97, 291-310.

Young, W. R. \& Jones, S. 1991 Shear dispersion. Phys. Fluids 3, 1087-1101. 